\title{
Secret Information Acquisition in Cournot Markets*
}

\author{
Esther Hauk \\ Department of Economics \\ Universidad Carlos III \\ Calle Madrid 126 \\ 28903 Getafe (Madrid) \\ Spain
}

\author{
Sjaak Hurkens \\ Department of Economics \\ Universitat Pompeu Fabra \\ Ramon Trias Fargas 25-27 \\ 08005 Barcelona \\ Spain
}

this version: August 1997
first version: March 1996

\begin{abstract}
Two-stage game models of information acquisition in stochastic oligopolies require the unrealistic assumption that firms observe the precision of information chosen by their competitors before determining quantities. This paper analyzes secret information acquisition as a one-stage game. Relative to the two-stage game firms are shown to acquire less information. Policy implications based on the two-stage game yield, therefore, too high taxes or too low subsidies for research activities. For the case of heterogeneous duopoly it is shown that comparative statics results partly depend on the observability assumption. Journal of Economic Literature Classification Numbers: C72, D43, D82.
\end{abstract}

Keywords: Information acquisition, oligopoly, uncertainty, Bayesian equilibrium.

We thank Antonio Cabrales and Fabrizio Zilibotti for helpful comments. Sjaak Hurkens gratefully acknowledges financial support from EC grant ERBCHGCT 93-0462. 


\section{Introduction}

Demand for a good is not deterministic but subject to stochastic shocks, that is, demand depends on some random variable. These shocks are usually modeled as uncertainty about the intercept of demand (as in this paper), the slope of the demand function, but more complex configurations are also possible. By doing market research, firms acquire information about this random variable. Having good information benefits firms since they will be able to predict better how large (residual) demand is, and therefore will make better production (or price) decisions which will result in higher profits (gross of the costs of information acquisition). Market research is also important for social welfare, because it allows demand and supply to be matched better. On the other hand, too much duplication of costly research is socially undesirable.

In most of the relevant literature information acquisition in stochastic oligopoly markets (and auctions) is modeled as a two-stage game. In the first stage the players decide how much to invest in information acquisition. In the second stage the choices made in the first stage become public and further decisions (quantities in oligopoly markets or bids in an auction) may therefore depend on these choices. In our view this "observability" assumption is unrealistic. How should a firm be able to observe the information acquisition decisions of its opponents? Disregarding espionage ${ }^{1}$, the only possibility seems to be that all firms publicly announce their information acquisition decisions. The only way to credibly announce the precision of information might be to make the reports, received from the market research agencies, public. However, firms often do not want to share their private information. (See e.g. Gal-Or (1985).) Of course, each firm will have some conjectures about how well informed the competitors are. And in equilibrium (of a one-stage game where information is gathered secretly) these beliefs will necessarily be confirmed. But this does not imply that the firm's quantity decision depends on the information acquisition decisions of the opponents.

Given the implausibility of the assumption that information acquisition decisions are observable, it is remarkable that the vast majority of the relevant literature has made this assumption without giving any justification. ${ }^{2}$ There could be two reasons for this. First, the analysis of the two-stage game seems to be easier since it can be solved by backward induction. The second stage game belongs to the class of games with imperfect (and asymmetric) information with which one is quite familiar by now. Plugging in the payoffs of the (unique) equilibria of the second stage games, the total game is reduced to one where players only decide how much information to acquire. However, solving the reduced game turns out to be not that straightforward after all. Checking the second

\footnotetext{
${ }^{1}$ Recall that information acquisition decisions in the two-stage game become public. In particular, each firm knows that its competitors know how well informed it is. This would imply that the presence of spies is also public knowledge.

${ }^{2}$ Information acquisition was studied in Cournot markets by Chang and Lee (1992), Daughety and Reinganum (1992), Hwang (1993, 1995), Li et al. (1987), Ockenfels (1989) and Vives (1988). Matthews (1984) and Milgrom (1981) studied information gathering in auctions. Only Matthews did not make the observability assumption, while Hwang (1995) stated that it would be desirable to analyze the game without this assumption. Hurkens and Vulkan (1995) considered information gathering by potential entrants and also criticized the observability assumption.
} 
order conditions is not an easy task. ${ }^{3}$ Moreover, explicit solutions could only be obtained for the limit case when the number of firms becomes infinite. We show that the one-stage game allows for explicit solutions for any number of firms.

A second reason for assuming observability of the information acquisition decision might have been that the authors were led to believe that the results do not depend on whether the assumption is made or not. In fact, Ponssard (1979, footnote 3) claims the latter to be the case in his oligopoly model where information is learned either perfectly or not at all. Although most authors did not model information acquisition as a binary choice, they may have thought that Ponssard's claim is valid also when the precision of information can be chosen from a continuum. However, even in the binary case Ponssard's claim is not completely correct. Section 2 contains a counterexample in which (for some parameter values) the one-stage game has more equilibria than the two-stage game.

When the precision of information can be chosen from a continuum the difference between the outcomes of the one and two-stage games is even more clear. We show that in finite Cournot markets firms will acquire less information if information is acquired secretly. This is the main result of the paper. The intuition is as follows. Firms gather information because they want to estimate residual demand. Raising the precision of information has two direct benefits, which are present in both models of information acquisition. It reduces the prediction errors of the intercept of demand and of the signals received by the competitors. Hence, both the demand curve and the production levels of the competitors can be estimated more accurately. In the two-stage model there is an additional benefit. If one firm raises its precision of information (in the first stage) other firms will react less aggressively to their own signal (in the second stage). This implies that the competitors' quantities will be predicted more precisely. Since the equilibrium level of information precision is found where marginal benefit equals marginal cost, it follows that firms invest more in research when they know that the competition will observe it (and respond to it).

The above argument reveals that in finite Cournot markets more information is acquired in the two-stage than in the one-stage game. This has an immediate consequence for policy implications concerning the need for taxes or subsidies on information gathering activities. Namely, policy advice based on the two-stage game will be biased in the direction of lower subsidies or higher taxes, compared to policy based on the (in our view) more realistic one-stage model. In fact, we show that even the direction of policy can be overturned: For some parameter constellations the one-stage game will advocate subsidies while the two-stage game will support taxes.

Since Vives (1988) and Li et al. (1987) were mainly interested in the competitive market, viewed as the limit of finite Cournot markets, it is interesting to analyze whether in the limit (when the number of firms goes to infinity) this difference disappears. It does. This is obvious if the competitive market with a continuum of firms is analysed directly: clearly the average precision of information in the market cannot be changed by an individual neglegible firm. However, when the competitive model is seen as the limit of finite Cournot markets, it is not obvious that both models of information acquisition

\footnotetext{
${ }^{3}$ This required about one page in both Vives (1988) and Li et al. (1987).
} 
yield the same results. As argued above, in each finite market firms' quantity decisions can be manipulated by a change in precision of information of a single firm. Even though the influence is small per opponent, the aggregate reaction of the market on a change in precision of information of one individual firm could be substantial also in the limit. That it is not does not justify the use of the two-stage model since most markets are finite.

The rest of the paper is organized as follows. The next section illustrates how important the assumption of observable information acquisition can be. We show this most convincingly in a simple example that is not related to oligopolistic markets. Then we show that Ponssard's (1979) claim that the two models of information acquisition are equivalent if information is either learned perfectly or not at all is wrong. In Section 3 a general model of information acquisition in an oligopolistic market with heterogeneous firms (of which Vives (1988), Li et al. (1987) and Hwang (1993) are special cases) is presented. We present our main result that firms acquire less information when information acquisition is not observed. We also show that equilibrium payoffs in the one-stage game are higher than in the two-stage game.

Section 4 analyzes the special case of homogeneous oligopoly and reconsiders the models of Vives (1988) and Li et al. (1987). We derive the explicit expression for the equilibrium amount of information gathering. Since we know that in the limit as the number of firms grows, the difference between secret and observable information acquisition disappears, we reinforce Vives'(1988) result, that competitive markets are second best efficient. In finite oligopolistic markets, however, firms may over- or underinvest in information acquisition. Conditions are stated under which firms underinvest relative to the social optimum. Finally, it is shown that policy implications derived from the different models of information acquisition may give qualitatively different results.

Section 5 concentrates on the special case of heterogeneous duopoly and reconsiders the model of Hwang (1993). Again we give the explicit expression for the equilibrium amount of information gathered and it is shown that firms will acquire less information when information is acquired secretly. We compare the comparative statics for the onestage game with those obtained by Hwang's (1993) for the two-stage game. In most cases the same conclusions are reached: (1) The firm with the higher marginal production cost will gather less information; (2) An increase in initial uncertainty and a decrease in the cost of information leads both firms to gather more information, but the firm with the higher marginal production cost increases information acquisition more; (3) An increase in the marginal production cost of one firm leads to a decrease of information acquisition of that firm and to an increase of information acquisition of its rival. However, our comparative statics results differ when considering changes in the slope of the demand function. This difference underlines once more the importance of modeling information acquisition in the right way.

Section 6 concludes and discusses some of the other models of information acquisition that have employed a two-stage model. 


\section{Secret vs. Private Information}

A very simple example (taken from Levine and Ponssard (1977)) illustrates how striking the difference between secret and private ${ }^{4}$ information acquisition can be. Suppose that Nature determines which of the two bimatrix games of Fig. 1 is going to be played, I or II. Game I is picked with probability $2 / 5$.

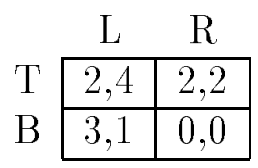

I

\begin{tabular}{l|c|c|}
\multicolumn{1}{c}{} & \multicolumn{1}{c}{$\mathrm{L}$} & \multicolumn{1}{c}{$\mathrm{R}$} \\
\cline { 3 - 3 } $\mathrm{T}$ & 2,2 & 2,4 \\
\cline { 2 - 3 } $\mathrm{B}$ & 0,0 & 3,1 \\
\cline { 2 - 3 } & &
\end{tabular}

II

Figure 1.

Only player 2 has the opportunity to learn the outcome of the random move. If neither player knows which game is played, both players have a dominant strategy and the outcome will be (T,R), yielding an expected payoff of $(2,16 / 5)$. However, if player 2 observes which matrix is chosen, he will play $\mathrm{L}$ in game I and $\mathrm{R}$ in game II. If player 1 knows that player 2 observes the outcome of the move of Nature, player 1 will play $\mathrm{B}$ and the resulting payoff vector will be $(3,1)$. In the two-stage version of the game of information acquisition player 2 will choose (commit) not to obtain information, and the outcome will be $(T, R)$. In the one-stage version, where information is acquired secretly, player 1 cannot condition his action on whether player 2 learned or not. In equilibrium player 1 will know that player 2 cannot resist to observe the outcome of the random move and therefore player 1 will choose B. Secret and private information acquisition lead to different outcomes.

The above example is of theoretical interest but does not have any economic content. Hypothetically, it could be that in examples of economic interest the difference between private and secret information acquisition is not important. Ponssard (1979) claims this to be the case in a linear model of quantity competition with $n$ firms and stochastic demand. The information acquisition decision is a binary one: one learns the true demand or one learns nothing. Ponssard computes the equilibria for each "subgame" where $k$ firms are informed and the remaining $n-k$ firms are uninformed. He shows that the uninformed firms always choose to produce the same quantity, independent of how many firms are informed, and that they always have the same expected payoff. This is caused by the fact that the expected quantity produced by an informed firm is independent of how many other firms are informed. From this he concludes that "... this theorem makes immaterial whether the acquisition of information is made privately or secretly". However, Ponssard has shown only that the incentives for an informed firm to deviate from any strategy profile are the same in the two different games, and that the incentives to deviate from the all-firms-stay-uninformed strategy profile are the

\footnotetext{
${ }^{4}$ This terminology is borrowed from Levine and Ponssard (1977).
} 
same in the two games. But he failed to examine the incentives for uninformed firms to deviate from any other strategy profile. The latter incentives do differ in the two games. Therefore, the solutions of the two games might not coincide.

To make the point more clear let us consider a very simple case. Consider a Cournot duopoly where price is given by $p=d-q$, where $q$ is the aggregate production and where $d$ is stochastic; with equal probability it is high $(h)$ or low $(l)$. Let $a=(l+h) / 2$ denote average demand. For convenience also assume that production is costless. Firms can learn the true realization of demand at cost $c$. First consider the two-stage game. If both firms learn the true demand they will produce $d / 3$. Expected payoff is therefore $\left(l^{2}+h^{2}\right) / 18=a^{2} / 9+\operatorname{Var}(d) / 9$. If both firms stay uninformed they will produce $a / 3$ resulting in an expected profit of $a^{2} / 9$. If only one firm learns the demand it is a little bit more complicated. The uninformed firm will produce $a / 3$. The informed firm will produce $(d-a / 3) / 2$. (Note that the expected production of the informed firm equals $a / 3$.) The expected profits are $a^{2} / 9$ for the uninformed firm and $a^{2} / 9+\operatorname{Var}(d) / 4$ for the informed firm. The pure equilibrium outcomes of the two-stage game are therefore: (1) if $c>\bar{c}:=\operatorname{Var}(d) / 4$ both firms stay uninformed; (2) if $c<\underline{c}:=\operatorname{Var}(d) / 9$ both firms become informed; $(3)$ if $c \in(\underline{c}, \bar{c})$ one firm becomes informed.

Now consider the one-stage game. Ponssard (1979) already has shown that the incentives to deviate for informed firms are the same. He also has shown that the incentives to deviate from the all-firms-stay-uninformed profile are the same. It follows that the situation where both firms become informed is an equilibrium outcome of the one-stage game if $c<\underline{c}$, and that the situation where both firms stay uninformed is an equilibrium outcome if $c>\bar{c}$. But the situation where only one firm becomes informed is an equilibrium outcome of the one-stage game if $c \in(\tilde{c}, \bar{c})$, where $\widetilde{c}:=\operatorname{Var}(d) / 16$. Namely, given the fact that the informed firm chooses $(d-a / 3) / 2$, the optimal deviation for the uninformed firm is to learn demand and produce $d / 2-(d-a / 3) / 4$ yielding a profit of $a^{2} / 9+\operatorname{Var}(d) / 16$. Hence, when the cost of becoming informed lies between $\tilde{c}$ and $\underline{c}$ the situation of asymmetrically informed firms is an equilibrium outcome of the one-stage game but not of the two-stage game. In the two-stage game the uninformed firm would deviate and learn the true demand. In this case an informed firm prefers the other firm to know that it is informed.

\section{The Model}

We will set up a model of information acquisition in Cournot markets which is very similar to Vives (1988). In fact, the present model will be slightly more general such that the models of Vives (1988), Li et al. (1987) and Hwang (1993) can be considered as special cases.

There are $n \geq 2$ firms. The inverse demand function is given by $p=\theta-\beta_{n} \sum_{j=1}^{n} x_{j}$, where $x_{j}$ is the output of firm $j, \beta_{n}>0$ is a constant and $\theta$ is a random parameter with mean $\mu$ and variance $\sigma^{2}$. Firm $i$ has a cost function $C_{i}\left(x_{i}\right)=c_{i} x_{i}+\lambda_{i} x_{i}^{2}$, where $c_{i} \geq 0$, $\lambda_{i} \geq 0$. Each firm $i$ can buy information of certain precision $1 / v_{i}$ about demand. This means that firm $i$ will receive a signal $s_{i}=\theta+\varepsilon_{i}$ where $\varepsilon_{i}$ is a noise term with zero mean, variance $v_{i}$ and with $\operatorname{Cov}\left(\theta, \varepsilon_{i}\right)=0$. The signals received by the firms are independent 
conditional on $\theta$ and furthermore it is assumed that $E\left(\theta \mid s_{i}\right)$ is affine in $s_{i}$. These assumptions imply that $E\left(\theta \mid s_{i}\right)=E\left(s_{j} \mid s_{i}\right)=\mu+t_{i}\left(s_{i}-\mu\right)$, where $t_{i}=\sigma^{2} /\left(\sigma^{2}+v_{i}\right)$. Note that as $v_{i}$ ranges from 0 to infinity, $t_{i}$ ranges from 1 to 0 . Instead of working with $v_{i}$, we shall work with $t_{i}$ and refer to $t_{i}$ as the precision of information. We assume that the cost of information acquisition is linear in $1 / v_{i}$, or equivalently, information of precision $t_{i}$ costs

$$
C\left(t_{i}\right)=\frac{c t_{i}}{\sigma^{2}\left(1-t_{i}\right)}
$$

A typical example of such an affine information structure is for $\theta$ and $\varepsilon_{i}$ to be Normal. $s_{i}$ could be the average of $n_{i}$ observations from a Normal distribution with mean $\theta$ and fixed variance. The precision of information $1 / v_{i}$ is proportional to the number of observations. When the marginal cost of an extra observation $c$ is constant, the cost of information will be linear in $1 / v_{i}$. (See Vives (1988) for a description of a number of other distributions that define an affine information structure.)

Let , 1 denote the one-stage game of information acquisition. A strategy for firm $i$ in this game is a pair $\left(t_{i}, x_{i}(\cdot)\right)$, where $t_{i} \in[0,1)$ is the choice of precision and where $x_{i}(\cdot)$ maps private signals into quantities. Let, 2 denote the two-stage game. In this game a strategy for firm $i$ is a pair $\left(t_{i}, y_{i}(\cdot, \cdot)\right)$, where $t_{i}$ again denotes the precision of information and where $y_{i}\left(t, s_{i}\right)$ denotes the quantity produced by $i$ in case it receives signal $s_{i}$ while firms have chosen to acquire information according to the tuple $t=\left(t_{1}, \cdots, t_{n}\right)$. Let , ${ }_{2}(t)$ denote the second stage continuation game of , 2 where precision tuple $t$ was chosen in the first stage. It is quite straightforward to solve for the Perfect Bayesian Nash equilibrium of , 2. First one solves for the (unique) Bayesian Nash equilibrium of , ${ }_{2}(t)$, for all $t$. Substitution of the equilibrium payoffs $\pi_{i}(t)-C\left(t_{i}\right)$ reduces the two-stage game to a game where only precision levels have to be chosen. This gives rise to reaction functions, and the equilibrium level of information is found by computing the intersection of these reaction functions, or, if the solution is interior, by equating the marginal value of information with its marginal cost:

$$
\frac{\partial \pi_{i}}{\partial t_{i}}=C^{\prime}\left(t_{i}\right)(\text { all } i)
$$

At first sight it seems that solving, ${ }_{1}$ will be more complicated. The precision of information $t_{i}$ and the action function $x_{i}(\cdot)$ have to be chosen simultaneously. Hence, it is not possible to work with reaction functions. However, a first order condition approach can be used. In fact, it will turn out that this is easier for the one-stage game than for the two-stage game. In particular, an explicit solution for , ${ }_{1}$ will be derived whereas the solution for , 2 can be given only implicitly. The key observation is that if $(t, x)$ is a pure Nash equilibrium of , ${ }_{1}$, it must be the case that $x$ is an equilibrium of,$_{2}(t)$. This reduces the number of candidate solutions of , ${ }_{1}$ considerably. Only these candidate solutions have to be checked against unilateral deviations $\left(t_{i}^{\prime}, x_{i}^{\prime}\right)$. 


\section{The Continuation Game}

As outlined above, the first step in the analysis of both , 1 and , 2 , focuses on the continuation games, ${ }_{2}(t)$. From the previous literature it is known that the equilibrium strategies in each continuation game are affine with respect to the signal. Instead of proving this here, we will impose that all quantity choice functions are affine.

Let $t=\left(t_{1}, \cdots, t_{n}\right)$ be a tuple of information precisions and consider the continuation game ${ }_{2}(t)$. Fix strategies $x_{j}\left(s_{j}\right)=a_{j}\left(s_{j}-\mu\right)+b_{j}$ for all $j \neq i$. The best reply for firm $i$ is that function $x_{i}(\cdot)$ that maximizes conditional expected profit

$$
E\left(x_{i}\left(s_{i}\right)\left(\theta-\beta_{n} \sum_{j \neq i} x_{j}\left(s_{j}\right)-c_{i}-\left(\lambda_{i}+\beta_{n}\right) x_{i}\left(s_{i}\right)\right) \mid s_{i}\right) .
$$

Hence,

$$
\begin{aligned}
x_{i}\left(s_{i}\right) & =\frac{E\left(\theta \mid s_{i}\right)-\beta_{n} \sum_{j \neq i} E\left(x_{j}\left(s_{j}\right) \mid s_{i}\right)-c_{i}}{2\left(\lambda_{i}+\beta_{n}\right)} \\
& =b_{i}+\bar{a}_{i} t_{i}\left(s_{i}-\mu\right),
\end{aligned}
$$

where

$$
b_{i}=\frac{\mu-c_{i}-\beta_{n} \sum_{j \neq i} b_{j}}{2\left(\lambda_{i}+\beta_{n}\right)} \quad \text { and } \quad \bar{a}_{i}=\frac{1-\beta_{n} \sum_{j \neq i} a_{j}}{2\left(\lambda_{i}+\beta_{n}\right)} .
$$

The conditional expected payoff (gross of information cost) from responding in this optimal way equals $\left(\lambda_{i}+\beta_{n}\right)\left(x_{i}\left(s_{i}\right)\right)^{2}$. The unconditional expected profit can now be computed to be

$$
\begin{aligned}
\pi_{i}(t) & :=E\left(\left(\lambda_{i}+\beta_{n}\right)\left(x_{i}\left(s_{i}\right)\right)^{2}\right) \\
& =\left(\lambda_{i}+\beta_{n}\right)\left(\left[E\left(x_{i}\left(s_{i}\right)\right)\right]^{2}+\operatorname{Var}\left(x_{i}\left(s_{i}\right)\right)\right) \\
& =\left(\lambda_{i}+\beta_{n}\right)\left(\bar{b}_{i}^{2}+\bar{a}_{i}^{2} t_{i}^{2}\left(\sigma^{2}+v_{i}\right)\right) \\
& =\left(\lambda_{i}+\beta_{n}\right)\left(\bar{b}_{i}^{2}+\bar{a}_{i}^{2} t_{i} \sigma^{2}\right) .
\end{aligned}
$$

Using (1) and (2) the equilibrium strategies can be computed and written as $x_{i}\left(s_{i}\right)=$ $b_{i}^{e}+\bar{a}_{i}^{e}[t] t_{i}\left(s_{i}-\mu\right)$, where

$$
2\left(\lambda_{i}+\beta_{n}\right) b_{i}^{e}=\mu-c_{i}-\beta_{n} \sum_{j \neq i} b_{j}^{e} \quad(i=1, \ldots, n)
$$

and

$$
2\left(\lambda_{i}+\beta_{n}\right) \bar{a}_{i}^{e}[t]=1-\beta_{n} \sum_{j \neq i} t_{j} \bar{a}_{j}^{e}[t] . \quad(i=1, \ldots, n)
$$

Note that the constants $b_{i}^{e}(i=1, \cdots, n)$ do not depend on the precision of information in the market.

Lemma 1 Solutions to systems (4) and (5) exist. Moreover, they are unique. 
Proof. See Appendix.

\section{Endogenous Information Acquisition}

The information acquisition games are now easily solved. We consider first the twostage game. The Perfect Bayesian equilibrium $\left(t^{*}, y^{*}(\cdot, \cdot)\right)$ of , 2 needs to be such that $y^{*}(t, \cdot)$ is the unique Nash equilibrium of ${ }_{2}(t)$ as computed above, for all $t$. Hence, $y_{i}^{*}\left(t, s_{i}\right)=b_{i}^{e}+a_{i}^{e}[t] t_{i}\left(s_{i}-\mu\right)$ for all $t$. Furthermore, no firm must have an incentive to gather any different amount of information. Assuming an interior solution this amounts to demanding that $\left(\partial \pi_{i} / \partial t_{i}\right)_{t^{*}}=C^{\prime}\left(t_{i}^{*}\right)$ or

$$
\left(\lambda_{i}+\beta_{n}\right) \sigma^{2}\left(\left(\bar{a}_{i}^{e}\left[t^{*}\right]\right)^{2}+2 t_{i}^{*} \bar{a}_{i}^{e}\left[t^{*}\right]\left(\frac{\partial \bar{a}_{i}^{e}}{\partial t_{i}}\right)_{\mid t^{*}}\right)=C^{\prime}\left(t_{i}^{*}\right) .
$$

It is not so easily verified that the second order condition is satisfied, but it can be done. (For the special cases of homogeneous firms and of heterogeneous duopoly see Vives (1988) and Hwang (1993), respectively.)

Consider now the one-stage game. In order for $(\bar{t}, \bar{x}(\cdot))$ to be a pure equilibrium of $,{ }_{1}, \bar{x}(\cdot)$ needs to be the equilibrium of,$_{2}(\bar{t})$. As before, this strategy profile can be computed and written as $x_{i}\left(s_{i}\right)=b_{i}^{e}+\bar{a}_{i}^{e}[\bar{t}] \bar{t}_{i}\left(s_{i}-\mu\right)$. The additional condition is that no firm must have an incentive to deviate from this profile. Note that firms can deviate from the information precision and the quantity decision function at the same time. However, given a deviation from $\bar{t}_{i}$ to $t_{i}$, the optimal deviation from $\bar{x}_{i}(\cdot)$ is easily seen to be $x_{i}\left(s_{i}\right)=b_{i}^{e}+\bar{a}_{i}^{e}[\bar{t}] t_{i}\left(s_{i}-\mu\right)$. This follows from (1) and (2). (Recall that the opponents do not observe the deviation and stick therefore to their strategies.) Note that $\bar{a}_{i}^{e}[\bar{t}]$ depends on $\bar{t}$, but not on $t_{i}$. Assuming an interior solution it follows from (3) that this amounts to demanding that

$$
\left(\lambda_{i}+\beta_{n}\right) \sigma^{2}\left(\bar{a}_{i}^{e}[\bar{t}]\right)^{2}=C^{\prime}\left(\bar{t}_{i}\right) .
$$

Here it is easily verified that the second order condition is satisfied, since $C^{\prime \prime}\left(t_{i}\right)>0$ and the left-hand side does not depend on $t_{i}$.

Comparing (6) and (7) it becomes obvious that the solutions of the two different information acquisition games do not coincide, as long as they are interior. In fact, whenever $\partial \bar{a}_{i}^{e}[t] / \partial t_{i}>0$ the solution of the two-stage game will yield higher levels of precision than the one-stage game. Indeed, we establish our main result:

Theorem 1 The equilibrium precisions of information in , 1 are strictly smaller than those in, 2 , unless they are zero in both.

Proof. From the equations (5) corresponding to firms $i$ and $j \neq i$ we deduce that

$$
2\left(\lambda_{i}+\beta_{n}\right) \bar{a}_{i}^{e}[t]+\beta_{n} t_{j} \bar{a}_{j}^{e}[t]=2\left(\lambda_{j}+\beta_{n}\right) \bar{a}_{j}^{e}[t]+\beta_{n} t_{i} \bar{a}_{i}^{e}[t] .
$$

It follows that $\bar{a}_{i}^{e}[t]>0$ for all $i$ in view of (5). Moreover, differentiating with respect to $t_{i}$ yields

$$
-\beta_{n} \bar{a}_{i}^{e}[t]+\left(2\left(\lambda_{i}+\beta_{n}\right)-t_{i} \beta_{n}\right) \frac{\partial \bar{a}_{i}^{e}[t]}{\partial t_{i}}=\left(2\left(\lambda_{j}+\beta_{n}\right)-t_{j} \beta_{n}\right) \frac{\partial \bar{a}_{j}^{e}[t]}{\partial t_{i}} .
$$


If it were true that $\frac{\partial \bar{a}_{i}^{e}[t]}{\partial t_{i}} \leq 0$, then $\frac{\partial \bar{a}_{j}^{e}[t]}{\partial t_{i}}<0$, for all $j \neq i$. This is impossible in view of equation (5). Therefore, $\frac{\partial \bar{a}_{i}^{e}[t]}{\partial t_{i}}>0$ and the result follows from inspection of equations (6) and (7).

Lemma 2 The equilibrium payoffs of, 2 are strictly lower than those of, 1 , unless no information is gathered in any of the games.

Proof. See Appendix.

Lemma 2 provides an argument why firms would not want to play the two-stage game even if they could choose to do so. Suppose firms acquire information secretly but some way exists to credibly inform the other firms of (a lower bound on) the precision of one's information. Given that each firm enjoys an additional benefit of information acquisition if the other firms are informed about the precision of its information, each firm would opt for the announcement. In equilibrium firms will acquire the level of information predicted by the two-stage game. Lemma 2 shows that the precision announcement game is a type of prisoner's dilemma: each firm prefers to gather more information and announce it, but when all firms do that, they are all worse off. Folk Theorem-type arguments support a no-announcement outcome in the infinitely repeated version of this game. Given the higher equilibrium payoffs of the one-stage game firms will consciously choose not to reveal the precision of their information.

\section{Homogeneous Firms}

In this section the special case of homogeneous firms will be considered. Let $\lambda_{i} \equiv \lambda$ and $c_{i} \equiv 0$ for all $i .{ }^{5}$ Given Lemma 1 it suffices to look for symmetric candidate solutions and unilateral deviations thereof. Let $\hat{t}$ denote a tuple of information precisions where $\hat{t}_{j} \equiv t^{*}$ for all $j \neq i$. Consider the equilibrium $x(\cdot)$ of,${ }_{2}(\hat{t})$. From $(4)$ and $(5)$ the equilibrium strategies can be computed. In particular, $x_{i}\left(s_{i}\right)=b_{i}^{e}+\bar{a}_{i}^{e}[\hat{t}] \hat{t}_{i}\left(s_{i}-\mu\right)$ where

$$
b_{i}^{e}=\mu /\left(2 \lambda+(n+1) \beta_{n}\right)
$$

and

$$
\bar{a}_{i}^{e}[\hat{t}]=\frac{2\left(\lambda+\beta_{n}\right)-\beta_{n} t^{*}}{2\left(\lambda+\beta_{n}\right)\left(2\left(\lambda+\beta_{n}\right)+\beta_{n}(n-2) t^{*}\right)-\beta_{n}^{2}(n-1) t^{*} \hat{t}_{i}} .
$$

It follows that

$$
a_{i}^{e}[\hat{t}]_{\mid \hat{t}_{j}=t^{*}}=1 /\left(2\left(\lambda+\beta_{n}\right)+(n-1) t^{*} \beta_{n}\right)
$$

and that

$$
\left(\frac{\partial \bar{a}_{i}^{e}[\hat{t}]}{\partial t_{i}}\right)_{\mid \hat{t}_{i}=t^{*}}=\frac{\beta_{n}^{2}(n-1) t^{*}}{\left(2\left(\lambda+\beta_{n}\right)-\beta_{n} t^{*}\right)\left(2\left(\lambda+\beta_{n}\right)+(n-1) \beta_{n} t^{*}\right)^{2}} .
$$

\footnotetext{
${ }^{5}$ The marginal cost parameter $c_{i}$ enters only in the constant term of the equilibrium strategies and does, therefore, not affect the results.
} 
Substitution of (9) and (10) in (6) and some further manipulations yield that the symmetric equilibrium precision of information $t^{*}$ is found by solving $M P V_{2}\left(t^{*}\right)=C^{\prime}\left(t^{*}\right)$, where

$$
M P V_{2}(t)=\sigma^{2}\left(\lambda+\beta_{n}\right) \frac{2\left(\lambda+\beta_{n}\right)(1+(n-1) \gamma)+(n-1) \gamma t \beta_{n}}{\left(2\left(\lambda+\beta_{n}\right)(1+(n-1) \gamma)-(n-1) \gamma t \beta_{n}\right)^{3}},
$$

if this solution is nonnegative. In this expression

$$
\gamma=\frac{t \beta_{n}}{2\left(\lambda+\beta_{n}\right)-t \beta_{n}}
$$

We use the notation $M P V_{2}(t)$ to denote the marginal private value to a firm of increasing its precision when all firms have acquired information of precision $t$. It is impossible to get an explicit solution for $t^{*}$. Only the limit solution for the case of infinitely many firms can be computed after taking the limit of $M P V_{2}$ as $n$ goes to infinity. This limit case will be of interest in order to compare our results with Vives (1988) and Li et al. (1987) who focused on this case.

Consider now the one-stage model. Substitution of (9) into (7) yields that the symmetric equilibrium precision of information $\bar{t}$ is found by solving $M P V_{1}(\bar{t})=C^{\prime}(\bar{t})$, where

$$
M P V_{1}(t)=\frac{\sigma^{2}\left(\lambda+\beta_{n}\right)}{\left(2\left(\lambda+\beta_{n}\right)+t(n-1) \beta_{n}\right)^{2}},
$$

as long as this solution is nonnegative. Here $M P V_{1}(t)$ denotes the marginal private value of information in the one-stage game. Using $C^{\prime}(t)=c /\left(\sigma^{2}(1-t)^{2}\right)$, the above expression can be solved explicitly to obtain:

$$
\bar{t}=\max \left\{0, \frac{\sigma^{2}-2 \sqrt{c\left(\lambda+\beta_{n}\right)}}{\sigma^{2}+(n-1) \beta_{n} \sqrt{c /\left(\lambda+\beta_{n}\right)}}\right\} .
$$

It is easily checked that $M P V_{2}(t)-M P V_{1}(t)>0$ for all $t>0$. To be precise,

$$
\frac{M P V_{2}(t)-M P V_{1}(t)}{\sigma^{2}\left(\lambda+\beta_{n}\right)}=\frac{2(n-1)\left(t \beta_{n}\right)^{2}}{\left(2\left(\lambda+\beta_{n}\right)-t \beta_{n}\right)\left(2\left(\lambda+\beta_{n}\right)+(n-1) t \beta_{n}\right)^{3}}
$$

The difference is proportional to (and of the same sign as) $\partial \bar{a}_{i} / \partial t_{i}$. (Compare (6) and (7).) Hence, $t^{*}>\bar{t}$, unless $t^{*}=\bar{t}=0$.

Note, however, that when $n$ tends to infinity $M P V_{2}(\cdot)$ and $M P V_{1}(\cdot)$ converge to the same function. Therefore, in the limit the difference between the outcomes of the two different information acquisition games disappears. This is independent of whether the market is replicated a la Vives (1988) or a la Li et al. (1987). In the model of Li et al. (1987) this result is not surprising. Since in their model $\beta_{n}=\beta$, which is independent of $n$, demand is not replicated when the number of firms grows. When $n$ goes to infinity, the gross profits per firm go to zero. Therefore, the amount of money spent on research has to go to zero. In the model of Vives (1988) where demand is replicated since $\beta_{n}=\beta / n$ the result is not at all obvious. In this case private information acquisition information has an additional benefit. If one firm raises its precision of information other firms will 
react less aggressively to their own signal. Therefore competitors' quantities will be predicted more precisely. This implies that in each finite Cournot market firms' quantity decisions can be manipulated by one single firm changing its information acquisition. When the number of firms grows, the influence per opponent diminishes. However, it is not obvious that the aggregate of these small influences is not substantial.

Note also that $M P V_{2}(0)=M P V_{1}(0)$ and that $M P V_{2}^{\prime}(0)=M P V_{1}^{\prime}(0)$. This implies that when the equilibrium amounts of information acquisition are close to zero (because information gathering is very costly or because initial uncertainty is quite small), then the two models predict approximately the same levels of information acquisition. For low information cost and high initial uncertainty the models will, however, predict very different levels of information gathering.

\section{Welfare}

Firms gather information in order to estimate residual demand and make higher profits. Consumers also benefit from the fact that demand and supply are matched better. When firms receive imprecise signals, some firms will overestimate demand while others will underestimate it. As a result firms will produce different quantities and, since production costs are convex, they will produce at different marginal costs, which clearly indicates an inefficiency. Better information reduces this inefficiency. On the other hand, a firm gathering information imposes a negative externality on its rivals. It raises its profits at the expense of the other firms. At high levels of information acquisition this lowers total industry profit. The duplication of market research by many firms also has a negative effect on social welfare.

The welfare aspects of information acquisition are therefore not clear and need to be examined. We need to define the efficient level of information and examine which policy measures are needed in order to obtain this optimal level. Since firms acquire more information in the two-stage game than in the one-stage game, policy implications are likely to differ with the model we use. Moreover, if our claim that the one-stage game is more realistic is true, it is important to understand how wrong policies based on the twostage game would be. Will policy implications be reversed, i.e. will the two-stage model recommend to tax (subsidize) information acquisition when it ought to be subsidized (taxed)? Or will it advocate a different magnitude of the same policy direction?

To address this issue three different definitions for the best (efficient) level of information will be examined that are characterized by a trade-off between efficiency and feasibility.

Def. 1 The first best (efficient) level of information is that level of information acquisition that maximizes welfare when firms use welfare maximizing quantities in production and the information of all firms can be pooled.

Vives (1988) has shown that (with strictly convex cost functions) the competitive market cannot attain the first best level of information, unless the cost of information is zero. There are simply no strategies that could yield the first best outcome, since convex costs imply that firms will surely operate at different marginal costs if they are to rely on their own private signal. With constant marginal cost, however, first best efficiency is possible. This result is opposed to the one of Li et al. (1987). The difference of results 
is caused by the fact that Li et al. (1987) do not replicate the market appropriately. Therefore, from now on we will only consider the properly replicated market, that is $\beta_{n}=\beta / n$.

The assumption that the information of all firms can be pooled is unrealistic. It does not respect the decentralized decision structure of the economy. Efficiency of competitive markets is restored if the constraint of decentralized information acquisition is recognized.

Def. 2 The second best (efficient) level of information is that level of information acquisition that maximizes welfare when firms use welfare maximizing quantity functions in production while information cannot be pooled.

Vives (1988) has shown that the competitive market attains this second best level. Since in the limit case firms acquire the same amount of information in the one-stage game of information acquisition as in the two-stage game, we get the following corollary to Vives' result:

Corollary 1 When the number of firms goes to infinity, the one-stage game model of information acquisition yields the second best efficient level of information.

The second best efficient level of welfare is problematic because it is based on firms maximizing welfare in production. It thereby implicitly assumes either a policy measure in the form of subsidizing production that induces firms to do so, or perfect competition. A subsidy on production is hard to implement since the size of the correct subsidy depends on the pool of information. The alternative implicit assumption of perfect competition makes the criterion inapplicable to finite oligopolistic markets. In perfectly competitive markets the second best efficient level of information coincides with the following criterion:

Def. 3 The third best (efficient) level of information is that level of information acquisition that maximizes welfare when firms use profit maximizing quantity functions in their production decision and information cannot be pooled.

Given that the third best efficient level of information respects the market structure in both information acquisition and production it seems the appropriate criterion to be used for policy recommendations. The first best level is irrelevant since firms can never be given incentives to pool their information. (See Gal-Or (1985).) Moreover, like the second best level it assumes some policy measure that ensures welfare maximization in production. Only the third measure concentrates on the pure effects of information acquisition and will therefore be the basis for our welfare analysis.

For given precision of information $t$ for each firm, each firm $j$ will use the equilibrium strategy $x_{j}\left(s_{j}\right)=a\left(s_{j}-\mu\right)+b \mu$ where $a$ and $b$ are determined by equations (8) and (9), respectively. Total welfare (gross of information cost) for given $t, \theta$ and signals $s_{j}$ equals

$$
T W\left(t, \theta, s_{1}, \ldots s_{n}\right)=\frac{\theta^{2}}{2 \beta_{n}}-\frac{1}{2 \beta_{n}}\left(\theta-\beta_{n} \sum_{j} x_{j}\left(s_{j}\right)\right)^{2}-\lambda \sum_{j} x_{j}\left(s_{j}\right)^{2} .
$$


We can compute the expected total welfare, $E T W(t)$ by first taking the expectation over signals conditional on $\theta$, and then taking the expectation over $\theta$. The third best efficient level $t_{e 3}$ satisfies $E T W^{\prime}\left(t_{e 3}\right)=n C^{\prime}\left(t_{e 3}\right)$, or equivalently,

$$
M S V\left(t_{e 3}\right)=C^{\prime}\left(t_{e 3}\right),
$$

where $M S V(t)=E T W^{\prime}(t) / n$ denotes the per capita marginal social value of information. This is equal to the marginal effect on total welfare when one firm increases its precision, when all firms have precision $t$. In the appendix we show that

$$
M S V(t)=\sigma^{2} \frac{2 \lambda^{2}+3 \beta_{n}^{2}+5 \beta_{n} \lambda+\lambda(n-1) t \beta_{n}+(n-1) t \beta_{n}^{2} / 2}{\left(2\left(\lambda+\beta_{n}\right)+(n-1) t \beta_{n}\right)^{3}} .
$$

We are now ready to compare the efficient level with the equilibrium level of information acquisition. Recall that at the equilibrium marginal private value equals marginal cost, while at the third best efficient level of information, the (per capita) marginal social value equals marginal cost. Whether under- or overinvestment takes place depends therefore on the relative positions of the curves $C^{\prime}, M S V$, and $M P V_{1}$ (for the one-stage game) and $M P V_{2}$ (for the two-stage game). We already know that $M P V_{1}$ lies below $M P V_{2}$ from Theorem 1 . The following lemma shows how the relative positions of the other curves exactly depend on the parameters of the model.

\section{Lemma 3}

(i) $M S V(t)>M P V_{1}(t)$ if and only if $t<\hat{t}_{1}$, where $\hat{t}_{1}=\frac{2+2 n \lambda / \beta}{n-1} . \hat{t}_{1}<1$ if and only if $n(1-2 \lambda / \beta)>3$.

(ii) $M S V(t)>M P V_{2}(t)$ if and only if $t<\hat{t}_{2}$, where $\hat{t}_{2}$ is the positive root of

$$
[(n-1)(2 n \lambda / \beta+3 / 2)] t^{2}+[n(n \lambda / \beta+1)] t-2(n \lambda / \beta+1)^{2}=0 .
$$

$\hat{t}_{2}<1$ if and only $\left(4(\lambda / \beta)^{2}-6 \lambda / \beta\right) n^{2}+(12 \lambda / \beta-5) n+7 \geq 0$.

Proof. See Appendix.

Lemma 3 tells us that the MSV and MPV curve intersect in a point $\hat{t}$ which depends on $\lambda / \beta$ and $n$. For $t<\hat{t}, M S V(t)>M P V(t)$ and for $t>\hat{t}$ the reverse holds. The reason is as follows. At low levels of information acquisition some firms under- and others overestimate demand considerably. This means that they will choose very different production levels, and since costs are convex, they will produce at different marginal costs, which indicates an inefficiency. (The inefficiency increases with $\lambda / \beta$.) Moreover, at low levels of information precision the negative externality that firms inflict on each other is smaller than at high levels. The marginal social value at low (high) levels of $t$ is therefore relatively high (low) compared to the marginal private value.

Note that $\hat{t}_{1} \geq 1$ when $\lambda / \beta \geq 1 / 2$ and that $\hat{t}_{2} \geq 1$ when $\lambda / \beta \geq 3 / 2$. Hence, when the inefficiency caused by firms producing at different marginal costs is high, the marginal social value is larger than the marginal private value, and as a consequence firms underinvest. This is true, whatever the size of the market, the cost of information gathering and the initial uncertainty. In this case subsidies on information acquisition activities could improve welfare. Note that the one-stage game model advocates higher subsidies than the two-stage model. 
When the inefficiency caused by firms producing at different marginal costs is not severe $(\lambda / \beta$ is low $)$, then for sufficiently large markets the intersection point of the marginal social value curve and the marginal private value curve lies within the interval $(0,1)$. Whether firms over- or underinvest now depends on the initial uncertainty and the cost of information acquisition. To be precise, it depends on the ratio $\sigma^{4} / c$. (See Lemma 7 in the Appendix.) Fig. 2 illustrates the three possible cases.

When information is cheap and initial uncertainty relatively large (see curve $C_{3}^{\prime}$ in Fig. 2), firms will overinvest. Taxes on information acquisition activities could restore this. (Note that the one-stage game calls for lower taxes than the two-stage model.) When information is expensive and initial uncertainty small (curve $C_{1}^{\prime}$ ), firms will underinvest relative to the optimum: subsidies are in order. (The one-stage game model calls for higher subsidies than the two-stage model.) Note that for intermediate values of the ratio $c / \sigma^{4}$ the one-stage game predicts underinvestment and calls for subsidies, while the two-stage game model predicts overinvestment and advocates taxes. (Curve $C_{2}^{\prime}$.)

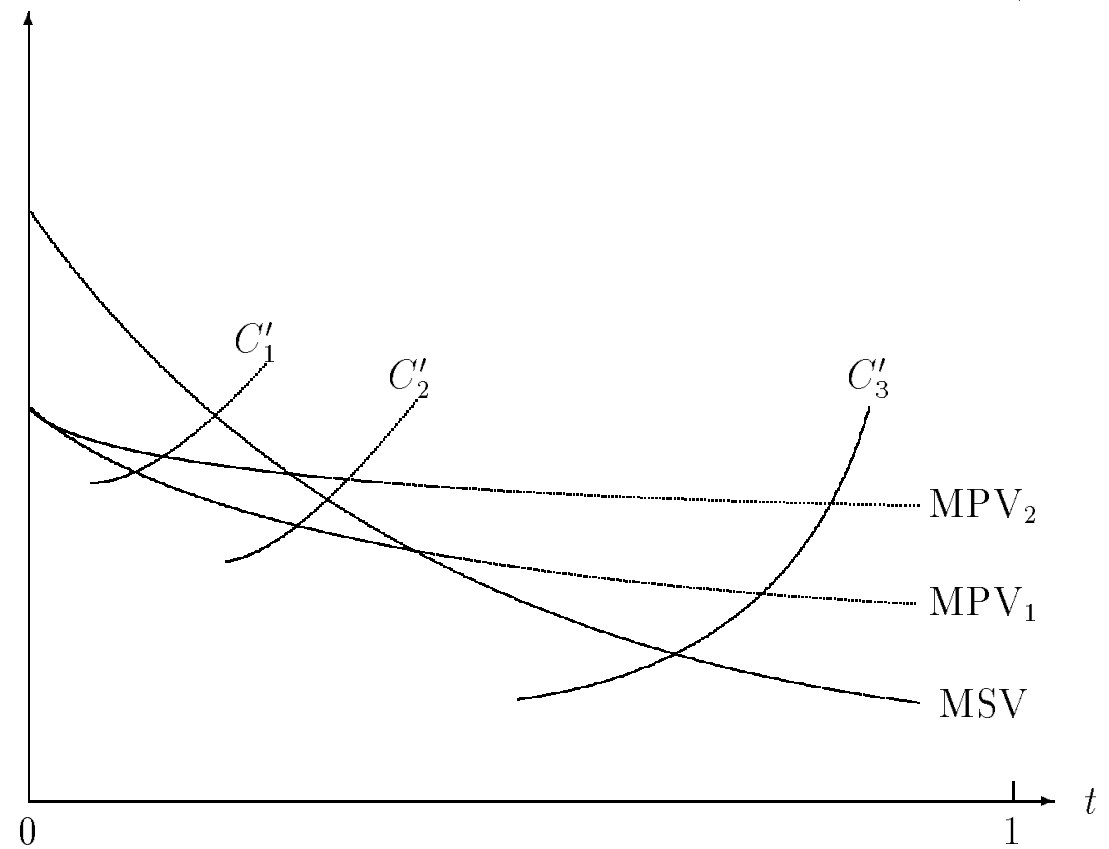

Figure 2: Comparing marginal cost and marginal values.

Note that $\sigma^{2}$ appears both in $M S V(t)$ and in $M P V(t)$ as a factor. Higher initial uncertainty amplifies the difference between the social and the private value, while it lowers and flattens $C^{\prime}(t)$. This means that for high initial uncertainty overinvestment will occur and that the introduction of the right tax could make up for a substantial welfare improvement. In this case the two different models of information acquisition would advocate very different tax levels and it is therefore important to use the relevant model. When initial uncertainty is very small, on the other hand, a small subsidy would be needed. The welfare improvement would not be very substantial in this case, and also the two different models of information acquisition would not yield very different policy recommendations. 
The above results show how the different parameters determine whether over- or underinvestment occurs. They also show that the two-stage model either advocates too low subsidies, too high taxes or a tax instead of a subsidy. In some circumstances the degree of over- or underinvestment is very small, in which case it does not really matter which model of information acquisition is used. This happens when $\beta \rightarrow 0, \sigma^{2} \rightarrow 0$ or $n \rightarrow \infty$. For $\beta \rightarrow \infty, \lambda \rightarrow \infty$ or $c \rightarrow \infty$ it is optimal not to acquire information and no policy measure is needed. In all other cases introducing the right policy measure can account for a substantial welfare improvement. In those cases it is important to use the right model, especially when initial uncertainty is large.

\section{$5 \quad$ Heterogeneous Duopoly}

For the case of heterogenous duopoly the general model of section 3 reduces to the model of Hwang (1993). We will show that the one-stage game admits an explicit solution whereas the two-stage game can only be solved implicitly. Moreover, we will show that the comparative statics results obtained by Hwang do not always coincide with the results we obtain for the one-stage game.

Let $n=2$ and write $\beta_{2}=\beta$. Let $t=\left(t_{i}, t_{j}\right)$ be a pair of information precisions. The equilibrium strategies of the continuation game, ${ }_{2}(t)$ can be computed using $(4)$ and (5). These computations show that

$$
x_{i}\left(s_{i}\right)=b_{i}^{e}+\bar{a}_{i}^{e}[t] t_{i}\left(s_{i}-\mu\right),
$$

where

$$
\begin{aligned}
\bar{a}_{i}^{e}[t] & =\frac{\left(2\left(\lambda_{j}+\beta\right)-\beta t_{j}\right)}{4\left(\lambda_{i}+\beta\right)\left(\lambda_{j}+\beta\right)-\beta^{2} t_{i} t_{j}} \\
b_{i}^{e} & =\frac{2\left(\lambda_{j}+\beta\right)\left(\mu-c_{i}\right)-\beta\left(\mu-c_{j}\right)}{4\left(\lambda_{i}+\beta\right)\left(\lambda_{j}+\beta\right)-\beta^{2}} .
\end{aligned}
$$

Differentiating (12) yields

$$
\frac{\partial \bar{a}_{i}^{e}}{\partial t_{i}}=\frac{\left(2\left(\lambda_{j}+\beta\right)-\beta t_{j}\right) \beta^{2} t_{j}}{\left(4\left(\lambda_{i}+\beta\right)\left(\lambda_{j}+\beta\right)-\beta^{2} t_{i} t_{j}\right)^{2}}
$$

Substituting (13) into (6) and assuming interior solutions one finds that the information precisions of the two-stage game $t_{i}^{*}(i=1,2)$ satisfy $\Delta_{i}\left(t^{*}\right)=C^{\prime}\left(t_{i}^{*}\right)(i=1,2)$ where

$$
\Delta_{i}(t)=\sigma^{2}\left(\lambda_{i}+\beta\right) \frac{\phi_{j}^{2}}{\psi^{3}}\left(\psi+2 t_{1} t_{2} \beta^{2}\right),
$$

where

$$
\phi_{j}=2\left(\lambda_{j}+\beta\right)-\beta t_{j} \text { and } \psi=4\left(\lambda_{i}+\beta\right)\left(\lambda_{j}+\beta\right)-\beta^{2} t_{i} t_{j} .
$$

As was the case for homogeneous firms, it is impossible to get explicit solutions for $t_{k}^{*}$ $(k=1,2)$. It is even impossible to compute the reaction function explicitly. Using the 
implicit function theorem one can prove that the reaction functions must be monotone decreasing. (See Hwang (1993).) Note that for $t_{i}^{*}<1$

$$
\frac{\left(1-t_{j}^{*}\right)^{2}}{\left(1-t_{i}^{*}\right)^{2}}=\frac{C^{\prime}\left(t_{i}^{*}\right)}{C^{\prime}\left(t_{j}^{*}\right)}=\frac{\left(\lambda_{i}+\beta\right)\left(2\left(\lambda_{j}+\beta\right)-\beta t_{j}^{*}\right)^{2}}{\left(\lambda_{j}+\beta\right)\left(2\left(\lambda_{i}+\beta\right)-\beta t_{i}^{*}\right)^{2}} .
$$

After taking square roots and defining $\rho_{k}=\sqrt{\lambda_{k}+\beta},(15)$ can be rewritten as $t_{j}^{*}=E_{j}\left(t_{i}^{*}\right)$ where

$$
E_{j}\left(t_{i}\right):=\frac{2 \rho_{i} \rho_{j}\left(\rho_{i}-\rho_{j}\right)+t_{i} \rho_{j}\left(2 \rho_{i} \rho_{j}-\beta\right)}{\rho_{i}\left(2 \rho_{i} \rho_{j}-\beta\right)+t_{i} \beta\left(\rho_{i}-\rho_{j}\right)}
$$

is defined for all $t_{i} \in[0,1]$. We call the curve defined by (16) the equilibrium curve.

The solution of the one-stage game is obtained by substituting (12) into (7). Some manipulations yield that the (interior) equilibrium precisions $\bar{t}_{i}(i=1,2)$ satisfy $\bar{\Delta}_{i}(\bar{t})=$ $C^{\prime}\left(\bar{t}_{i}\right)(i=1,2)$ where

$$
\bar{\Delta}_{i}(t)=\sigma^{2}\left(\lambda_{i}+\beta\right) \frac{\phi_{j}^{2}}{\psi^{2}}
$$

Note that

$$
\frac{\left(1-\bar{t}_{j}\right)^{2}}{\left(1-\bar{t}_{i}\right)^{2}}=\frac{C^{\prime}\left(\bar{t}_{i}\right)}{C^{\prime}\left(\bar{t}_{j}\right)}=\frac{\left(\lambda_{i}+\beta\right)\left(2\left(\lambda_{j}+\beta\right)-\beta \bar{t}_{j}\right)^{2}}{\left(\lambda_{j}+\beta\right)\left(2\left(\lambda_{i}+\beta\right)-\beta \bar{t}_{i}\right)^{2}} .
$$

Hence $\bar{t}_{j}=E_{j}\left(\bar{t}_{i}\right)$. The solutions of the two different games of information acquisition lie on the same equilibrium curve. It is easily seen that for $t_{1} t_{2}>0 \Delta_{i}(t)>\bar{\Delta}_{i}(t)$ and that, therefore, $t_{i}^{*}>\bar{t}_{i}$ for $i=1,2$ whenever the solutions are interior.

From (17) we obtain

$$
\sigma^{2} \sqrt{\lambda_{i}+\beta}\left(2\left(\lambda_{i}+\beta\right)-\beta t_{j}\right)\left(1-t_{i}\right)=\sqrt{c}\left(4\left(\lambda_{i}+\beta\right)\left(\lambda_{j}+\beta\right)-\beta^{2} t_{i} t_{j}\right)
$$

which can be rewritten as

$$
t_{j}\left(t_{i} \sqrt{c} \beta^{2}-\beta \sigma^{2} \rho_{i}\left(1-t_{i}\right)\right)=4 \rho_{i}^{2} \rho_{j}^{2} \sqrt{c}-2 \sigma^{2} \rho_{i} \rho_{j}^{2}\left(1-t_{i}\right) .
$$

Since we are interested in the case where $t_{i}, t_{j} \leq 1$ we have $\bar{t}_{j}=R_{j}\left(\bar{t}_{i}\right)$ where

$$
R_{j}\left(t_{i}\right):=\frac{4 \rho_{i}^{2} \rho_{j}^{2} \sqrt{c}-2 \sigma^{2} \rho_{i} \rho_{j}^{2}\left(1-t_{i}\right)}{t_{i} \sqrt{c} \beta^{2}-\beta \sigma^{2} \rho_{i}\left(1-t_{i}\right)}
$$

is defined for $t_{i} \in\left[0, \sigma^{2} \rho_{i} /\left(\beta \sqrt{c}+\sigma^{2} \rho_{i}\right)\right)$. We will refer to $R_{j}$ as firm $j$ 's pseudo reaction curve. It is of course not really a reaction curve because a strategy for each firm is a pair $(t, x(\cdot))$ where $t$ is the precision of information and where $x(\cdot)$ is a mapping that assigns to each signal a quantity. Still, the equilibrium values $\bar{t}_{i}$ and $\bar{t}_{j}$ are found by computing the intersection point of the pseudo reaction curves, or alternatively, by computing the intersection point of $R_{j}$ and $E_{j}$.

Note that $R_{j}$ is downward sloping and has a vertical asymptote, while $E_{j}$ is upward sloping. Without loss of generality we may assume that $\lambda_{i} \geq \lambda_{j}$ which implies that $E_{j}(0) \geq 0$. The necessary and sufficient condition for the existence of a unique and interior solution is therefore $R_{j}(0)>E_{j}(0)$. Hence, 
Lemma 4 Suppose $\lambda_{i} \geq \lambda_{j}$. The sufficient and necessary condition for the existence of a unique interior equilibrium is

$$
\sqrt{c}<\frac{\sigma^{2}\left(2 \rho_{j}^{2}-\beta\right)}{2 \rho_{j}\left(2 \rho_{i} \rho_{j}-\beta\right)} .
$$

Proof: Straightforward calculations.

Assuming that an interior solution exists, we can explicitly compute it by computing the intersection of the equilibrium curve and the pseudo reaction curve. We obtain

Lemma 5 Suppose the equilibrium levels of information acquisition are strictly positive. Then

$$
\bar{t}_{i}=\frac{\sigma^{2} \rho_{i}\left(2 \rho_{j}^{2}-\beta\right)-2 \rho_{i} \rho_{j}\left(2 \rho_{i} \rho_{j}-\beta\right) \sqrt{c}}{\sigma^{2} \rho_{i}\left(2 \rho_{j}^{2}-\beta\right)+\beta\left(2 \rho_{i} \rho_{j}-\beta\right) \sqrt{c}} .
$$

Proof: Straightforward calculations.

We will use the equilibrium curve and the pseudo reaction curves to obtain some comparative statics results, and will compare them to those obtained by Hwang (1993) for the two-stage game.

Lemma 6 Let $\lambda_{i}>\lambda_{j}$.

(i) $E_{j}\left(t_{i}\right)$ is increasing and concave and has slope smaller than 1

(ii) $E_{j}\left(t_{i}\right)$ is independent of $c$ and $\sigma^{2}$.

(iii) $R_{j}\left(t_{i}\right)$ is decreasing.

(iv) $R_{j}\left(t_{i}\right)$ shifts downward when $c$ or $\lambda_{i}$ increase and when $\lambda_{j}$ or $\sigma^{2}$ decrease.

(v) $R_{i}\left(t_{j}\right)$ shifts downward when $\lambda_{i}$ decreases and when $\lambda_{j}$ increases.

(vi) $R_{j}\left(t_{i}\right)$ shifts downward when $\beta$ increases.

(vii) The equilibrium curve $E_{j}(t)$ shifts downward when $\beta$ increases.

Proof: See Appendix.

Lemma 6 allows us to draw the following conclusions:

1. From the fact that $E_{j}$ is concave, $E_{j}(0) \geq 0$, and $E_{j}(1)=1$ it follows that $\bar{t}_{j} \geq \bar{t}_{i}$ where equality holds if and only if $\lambda_{i}=\lambda_{j}$. That is, the firm with the lower slope of the marginal cost function will acquire more information.

2. As the pseudo reaction curve shifts upwards when $\sigma^{2}$ increases and downwards when $c$ increases while the equilibrium curve is unaffected by these parameters, an increase in initial uncertainty $\sigma^{2}$ or a decrease in information gathering cost $c$ leads both firms to gather more information. Since $E_{j}$ is concave and $E_{j}^{\prime}(0) \leq 1$ we have $E_{j}^{\prime}\left(t_{i}\right) \leq 1$ with equality only in the case of identical firms. We may now conclude that firm $i$ (recall $\lambda_{i} \geq \lambda_{j}$ ) increases information acquisition more than firm $j$ when initial uncertainty increases or information gathering cost decreases, both in absolute terms and in relative terms. This situation is illustrated in Fig. 3. 


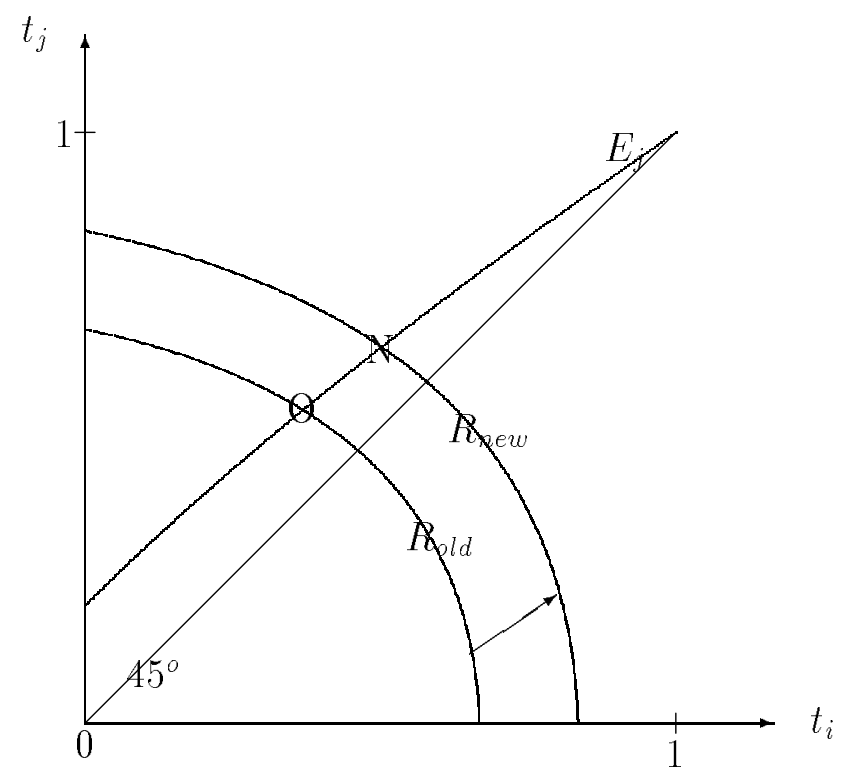

Figure 3: effect of increase in $\sigma^{2}$ or decrease in $c$

3. As $\lambda_{i}$ increases, $R_{j}$ shifts downward and $R_{i}$ upward. Since $R_{i}$ is flatter than $R_{j}{ }^{6}$, $\bar{t}_{i}$ decreases and $\bar{t}_{j}$ increases which is illustrated in Fig. 4.

${ }^{6}$ This follows from the sufficient and necessary condition for the existence of a unique interior equilibrium (Lemma 4). 


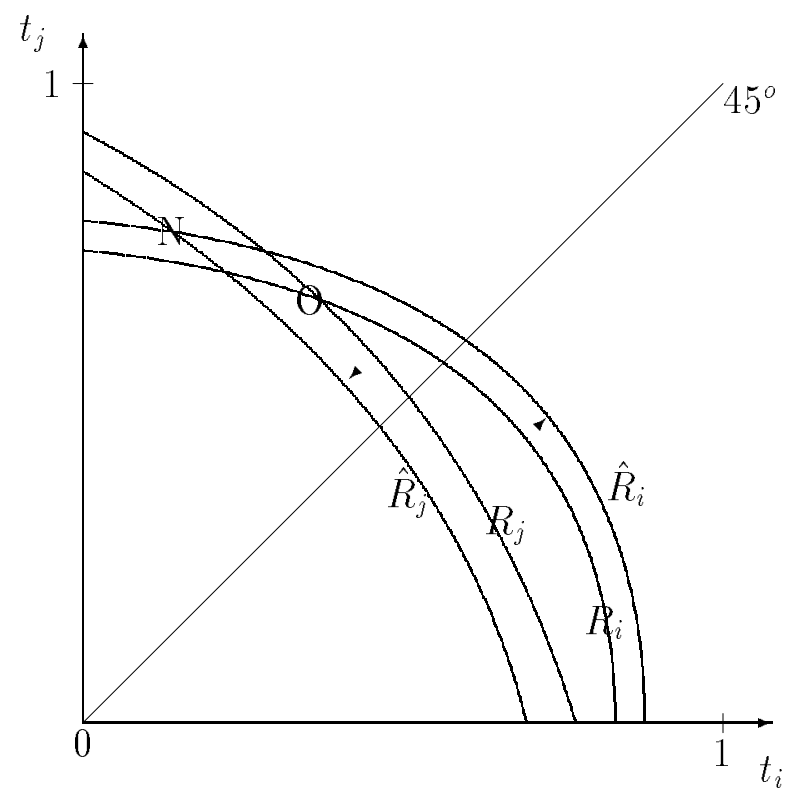

Figure 4: An increase in $\lambda_{i}$ shifts $R_{i}$ up and $R_{j}$ down.

4. Similarly, an increase in $\lambda_{j}$ leads to an increase in $\bar{t}_{i}$ and a decrease in $\bar{t}_{j}$.

5. How is the information acquisition affected when $\beta$ increases? $R_{j}$ shifts downward when $\beta$ increases. The equilibrium curve $E_{j}(t)$ shifts downward when $\beta$ increases (for $t \in[0,1]$ and when $\lambda_{i}>\lambda_{j}$ ). It follows that an increase in $\beta$ results in a decrease in $t_{j}$. From the direction of movements of the curves alone, however, the effect on $t_{i}$ cannot be determined. It will depend on the relative movements of the curves. Fig. 5 illustrates the two possible effects. 


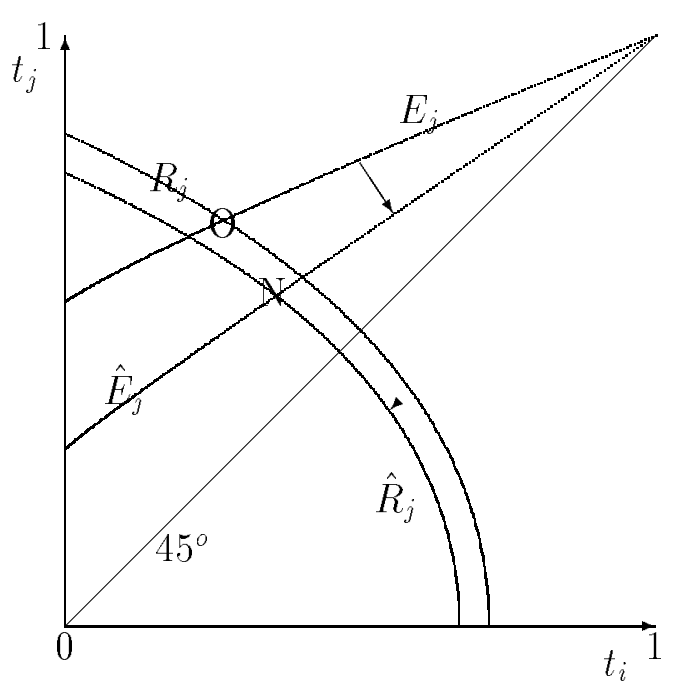

Fig. 5a: $t_{i}$ increases when $\beta$ increases

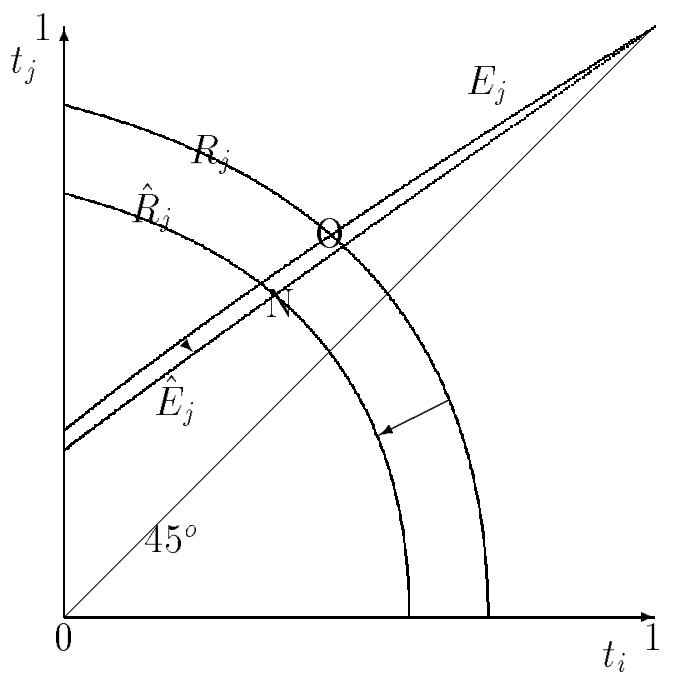

Fig. 5b: $t_{i}$ decreases when $\beta$ increases

Figure 5: An increase of $\beta$ has ambiguous effects

In order to determine the effect on $t_{i}$ we need to use the exact expression for $\bar{t}_{i}$ presented in Lemma 5. It turns out that we can find parameter values that show that the effect of an increase in $\beta$ on $t_{i}$ is indeed ambiguous ${ }^{7}$. This is in sharp contrast with the comparative statics results of Hwang's two-stage model, where an increase in $\beta$ unambiguously leads to a decrease in $t_{i}$. Thus besides yielding different levels of information acquisitions, which in contrast to Hwang can be calculated explicitly, the one-stage model differs from the two-stage model in the case of heterogeneous duopoly in the way firms adjust to a change in $\beta$. This difference underlines the importance of the way in which information acquisition is modeled.

\section{Conclusions}

In an oligopoly market with uncertain demand firms acquire information in order to estimate (residual) demand. When information acquisition is modeled as a two-stage game, as has been customary, there is an additional but unrealistic benefit to obtaining information. If one firm is known to have good information, other firms will act less aggressively towards their own private information and this makes their behavior easier to predict. In these two-stage models firms therefore overinvest in market research relative to the case of secret information acquisition. It was shown, that this overinvestment

\footnotetext{
${ }^{7}$ For example keeping $\sigma^{2}=1, \sqrt{c}=0.001979, \lambda_{i}=15796.4, \lambda_{j}=0.001$ and changing $\beta$ from 1 to 3000 will lead to an increase of $t_{i}$ from 0.01 to 0.112 , while increasing $\beta$ further to 9000 will cause a drop in $t_{i}$ to 0.102 .
} 
vanishes when the number of firms becomes very large. This implies that in a very competitive market the second (third) best efficient level of information is acquired. In smaller markets firms may under- or overinvest with respect to the efficient level of information acquisition. Policy implications depend always quantitatively on which model of information acquisition is considered. In some instances the policies advocated by the two models are even qualitatively different (tax versus subsidy). Using the "right" model is therefore important.

In this paper it was argued that the one-stage game is the more relevant model since firms are not able to observe the information acquisition decisions of their opponents. The two-stage game would be appropriate if firms could credibly commit to (a lower bound on) the precision of information and would deliberately chose to do so. However, Lemma 2 showed that firms are better off not announcing (a lower bound on) the precision of their information.

This paper reconsidered the models of Ponssard (1979), Li et al. (1987), Vives (1988) and Hwang (1993) in detail. There are some other models of information acquisition that have not been discussed yet. Ockenfels (1989) considers a model very similar to the one of Ponssard (1979). The only difference is that in Ockenfels' model quantity choices are discrete (in fact binary). It is clear that his model exhibits the same problem as Ponssard (1979).

Chang and Lee (1992) discuss a model of differentiated duopoly which did not fit nicely in the model presented in section 3, although the present model could be extended to include differentiated products as well. Again information acquisition is modeled as a two-stage game. It can be easily verified by computing the best reply against an affine strategy, as was done in section 3 , that also in their model firms overinvest in research relative to the case of secret information acquisition.

Hwang (1995) considers a model of information acquisition that is designed to compare monopoly, duopoly, and competitive markets. There are only two players in the model. The second stage game is modeled using conjectural variations. By varying the conjectural variations the model can represent monopoly, duopoly or a competitive market. However, in the first stage there are no conjectural variations. Hence, the influence of raising the precision of information are more or less the same as in the ordinary duopoly game. This means that firms overinvest in research in Hwang's (1995) model even in the case of a competitive market. The peculiarity of this model is further illustrated by Lemma 4 in Hwang (1995). It says that the level of information precision that maximizes joint profit is smaller than the equilibrium precision. The Lemma is mathematically correct, but does not make any sense in the case the model is to represent a monopoly.

Further models of information acquisition have been studied for auctions. Milgrom (1981) considers a two-stage version whereas Matthews (1984) considers the one-stage version. Unfortunately Matthews (1984) was unable to get an explicit solution. Further research has to be conducted for the case of auctions. One should note, though, that the main interest in the literature on information acquisition in auctions is when the number of bidders becomes very large. The question addressed is whether the winning bid will converge (with probability one) to the true value of the object. Because of the similarity with competitive markets one might conjecture that it does not matter 
whether information is acquired secretly or not. However, this needs to be examined carefully.

\section{Appendix}

\subsection{Existence of equilibrium}

Proof of Lemma 1.

Let $\mathrm{A}$ denote the $n \times n$ matrix with entries $A_{i i}=2\left(\lambda_{i}+\beta_{n}\right)(i=1, \ldots, n)$ and $A_{i j}=\beta_{n} t_{j}(i \neq j)$. Existence and uniqueness of solutions for equations (4) and (5) is equivalent to showing that $\mathrm{A}$ is non-singular. Subtracting the $j$-th row from the $j+1$-st row $(j=n-1, \ldots, 1)$ yields a matrix $\mathrm{B}$ with the same determinant as $\mathrm{A}$ which has zeros everywhere, except in the first row and in the entries $(i, j)$ where $i=j>1$ $\left(B_{j j}=2\left(\lambda_{j}+\beta_{n}\right)-\beta_{n} t_{j}\right)$ or $i=j+1>1\left(B_{j+1, j}=\beta_{n} t_{j}-2\left(\lambda_{j}+\beta_{n}\right)\right)$. Subtracting $B_{k+1, k} / B_{k+1, k+1}$ times the $(k+1)$-st column from the $k$-th column (for $k=n-1, \ldots, 1$ ) yields a matrix $C$ with the same determinant as $B$ with zeros everywhere, except in the first row and the diagonal. The determinant of $\mathrm{C}$ is thus egual to the product of its diagonal elements, which is not equal to zero since $C_{i i}=B_{i i}>0$ for $i>1$ and $C_{11}=A_{11}-\beta_{n} t_{2}\left(\beta_{n} t_{1}-2\left(\lambda_{1}+\beta_{n}\right)\right) /\left(2\left(\lambda_{2}+\beta_{n}\right)-\beta_{n} t_{2}\right)>0$, where the last inequality follows from the fact that $t_{1} t_{2} \leq 1$ and $\lambda_{1}, \lambda_{2}, \beta_{n}>0$.

\subsection{Equilibrium payoffs}

Proof of Lemma 2.

Recall that $\bar{t}$ (resp. $t^{*}$ ) denote the equilibrium level of information acquisition in the one-stage game (resp. two-stage game), and that $\bar{t}<t^{*}$. Let $\bar{a}^{e}[t]=1 /\left(2\left(\lambda+\beta_{n}\right)+(n-\right.$ 1) $\left.t \beta_{n}\right)$ and $b^{e}=\mu /\left(2 \lambda+(n+1) \beta_{n}\right)$, such that $x(s)=b^{e} \mu+\bar{a}^{e}[t] t(s-\mu)$ is the equilibrium strategy of each firm in the second stage game, $(t, \ldots, t)$. Using $(3)$ we know that the equilibrium payoff in this second stage game equals

$$
\pi(t)=\left(\lambda+\beta_{n}\right)\left(\left(b^{e}\right)^{2}+\left(\bar{a}^{e}[t]\right)^{2} t \sigma^{2} .\right.
$$

The equilibrium payoffs for the one- and two-stage game are therefore $\pi(\bar{t})-C(\bar{t})$ and $\pi\left(t^{*}\right)-C\left(t^{*}\right)$. Now

$$
\begin{aligned}
\pi^{\prime}(t)-C^{\prime}(t) & =\sigma^{2}\left(\lambda+\beta_{n}\right) \frac{2\left(\lambda+\beta_{n}\right)-(n-1) \beta_{n} t}{\left(2\left(\lambda+\beta_{n}\right)+(n-1) \beta_{n} t\right)^{3}}-C^{\prime}(t) \\
& <M P V_{1}(t)-C^{\prime}(t)
\end{aligned}
$$

where $M P V_{1}(t)$ is the marginal private value of information acquitision in the onestage game defined by 4 . The right-hand side of (20) is negative for $t>\bar{t}$. Hence, $\pi(\bar{t})-C(\bar{t})>\pi\left(t^{*}\right)-C\left(t^{*}\right)$. 


\subsection{The third best level of information}

We assume that the solution is symmetric. For given precision of information $t$ for each firm, each firm $j$ will use strategy $x_{j}\left(s_{j}\right)=a\left(s_{j}-\mu\right)+b \mu$ where $a$ and $b$ are the equilibrium strategies as computed in Section 4, i.e.

$$
b=1 /\left(2 \lambda+(n+1) \beta_{n}\right),
$$

and

$$
a=t /\left(2\left(\lambda+\beta_{n}\right)+(n-1) \beta_{n} t\right) .
$$

Total welfare, gross of information costs, equals for given precision $t$ and fixed $\theta$ and fixed signals $s_{j}$

$$
T W\left(t, \theta, s_{1}, \ldots s_{n}\right)=\frac{\theta^{2}}{2 \beta_{n}}-\frac{1}{2 \beta_{n}}\left(\theta-\beta_{n} \sum_{j} x_{j}\left(s_{j}\right)\right)^{2}-\lambda \sum_{j} x_{j}\left(s_{j}\right)^{2} .
$$

Expected total welfare, given $\theta$ equals

$$
\begin{aligned}
E(T W \mid \theta)= & \frac{\theta^{2}}{2 \beta_{n}}-\frac{1}{2 \beta_{n}}\left\{\left[E\left(\left(\theta-\beta_{n} \sum a\left(s_{j}-\mu\right)+b \mu\right) \mid \theta\right)\right]^{2}+\right. \\
& \left.+\operatorname{Var}\left(\left(\theta-\beta_{n} \sum a\left(s_{j}-\mu\right)+b \mu\right) \mid \theta\right)\right\}+ \\
& -\lambda \sum\left[E\left(a\left(s_{j}-\mu\right)+b \mu \mid \theta\right)^{2}+\operatorname{Var}\left(a\left(s_{j}-\mu\right)+b \mu \mid \theta\right)\right]= \\
= & \frac{\theta^{2}}{2 \beta_{n}}-\frac{1}{2 \beta_{n}}\left[\theta-\beta_{n} n(a(\theta-\mu)+b \mu)\right]^{2}+ \\
& -\frac{1}{2 \beta_{n}} \beta_{n}^{2} n a^{2} v-\lambda n(a(\theta-\mu)+b \mu)^{2}-\lambda n a^{2} v .
\end{aligned}
$$

Taking the expectation over $\theta$ gives unconditional expected welfare

$$
\begin{aligned}
\operatorname{ETW}(t)= & \frac{\mu^{2}+\sigma^{2}}{2 \beta_{n}}-\frac{1}{2 \beta_{n}}\left(\mu-\beta_{n} n(a(\mu-\mu)+b \mu)\right)^{2}+ \\
& -\frac{1}{2 \beta_{n}}\left(1-\beta_{n} n a\right)^{2} \sigma^{2}+ \\
& -\frac{1}{2} \beta_{n} n a^{2} v-\lambda n a^{2} \sigma^{2}-\lambda n(b \mu)^{2}-\lambda n a^{2} v .
\end{aligned}
$$

At the optimal level of information acquisition, $t_{e 3}$, we have $E T W^{\prime}\left(t_{e 3}\right)=n C^{\prime}\left(t_{e 3}\right)$. Define the (per capita) marginal social value of information as

$$
M S V(t)=E T W^{\prime}(t) / n
$$

Now it is straightforward to check that

$$
\begin{aligned}
M S V(t)= & a^{\prime} \sigma^{2}-n \beta_{n} a a^{\prime} \sigma^{2}-\beta_{n} a a^{\prime} v-\beta_{n} a^{2} v^{\prime} / 2 \\
& -2 \lambda a a^{\prime} \sigma^{2}-2 \lambda a a^{\prime} v-\lambda a^{2} v^{\prime} \\
= & \sigma^{2} \frac{2 \lambda^{2}+3 \beta_{n}^{2}+5 \beta_{n} \lambda+\lambda(n-1) t \beta_{n}+(n-1) t \beta_{n}^{2} / 2}{\left(2\left(\lambda+\beta_{n}\right)+(n-1) t \beta_{n}\right)^{3}} .
\end{aligned}
$$




\subsection{Welfare analysis}

Proof of Lemma 3.

(i) It is easily verified that $M S V(t)>M P V_{1}(t)$ if and only if

$$
\lambda \beta_{n}+\beta_{n}^{2}-(n-1) t \frac{\beta_{n}^{2}}{2}>0 .
$$

In particular, $M S V(0)>M P V_{1}(0)$. Furthermore, the two curves intersect at $\hat{t}_{1}=$ $2(n \lambda / \beta+1) /(n-1) . \hat{t}_{1} \leq 1$ if and only if $n(1-2 \lambda / \beta) \geq 3$. Obviously, when $2 \lambda / \beta \geq 1$, no $n$ exists for which the inequality holds. On the other hand, if $2 \lambda / \beta<1$, the inequality holds for large enough $n$.

(ii) It is easily verified that $M S V(t)>M P V_{2}(t)$ if and only if

$$
(n-1)\left[t \beta_{n}\left(\lambda+\beta_{n}\right)+t^{2} \beta_{n}\left(2 \lambda+\frac{3}{2} \beta_{n}\right)\right]-\left(\lambda+\beta_{n}\right)\left[\left(2\left(\lambda+\beta_{n}\right)-t \beta_{n}\right]<0 .\right.
$$

In particular, $M S V(0)>M P V_{2}(0)$. Furthermore, the two curves intersect only once in the halfline $[0, \infty)$, namely in $\hat{t}_{2}$, the positive root of the equation mentioned in the statement of the Lemma.

$$
\hat{t}_{2}=\frac{(n \lambda / \beta+1)\left(-n+\sqrt{n^{2}+8(n-1)(2 n \lambda / \beta+3 / 2)}\right)}{2(n-1)(2 n \lambda / \beta+3 / 2)}
$$

This intersection point lies in the interval $[0,1]$ only if the left-hand side of the above inequality, evaluated at $t=1$, is positive, i.e. if

$$
(n-1)(6 n \lambda / \beta+5) \geq 2(n \lambda / \beta+1)(2 n \lambda / \beta+1) .
$$

This is equivalent to

$$
n^{2}\left(6 \lambda / \beta-4(\lambda / \beta)^{2}\right)+n(5-12 \lambda / \beta)-7 \geq 0 .
$$

Obviously, when $\lambda / \beta \geq 3 / 2$, there exists no $n>0$ for which the above inequality holds. If $\lambda / \beta<3 / 2$, for large enough $n$ the inequality is satisfied.

\section{Lemma 7}

(i) Suppose that $\lambda, \beta$ and $n$ are such that $\hat{t}_{1}<1$, i.e. the marginal social value curve intersects the marginal private value curve in the interval $(0,1)$. Then there exists some treshold $x_{1}$ (which depends on $\lambda, \beta$ and $n$ ) such that overinvestment occurs if and only if $\sigma^{4} / c>x_{1}$.

(ii) Suppose that $\lambda, \beta$ and $n$ are such that $\hat{t}_{2}<1$, i.e. the marginal social value curve intersects the marginal private value curve in the interval $(0,1)$. Then there exists some treshold $x_{2}$ (which depends on $\lambda, \beta$ and $n$ ) such that overinvestment occurs if and only if $\sigma^{4} / c>x_{2}$. 
Proof: (i) Suppose that $M S V$ and $M P V_{1}$ intersect in $\hat{t}_{1}<1$. Overinvestment occurs when $\bar{t}>\hat{t}_{1}$ or, equivalently, when $M P V_{1}\left(\hat{t}_{1}\right)>C^{\prime}\left(\hat{t}_{1}\right)$. Hence, overinvestment occurs if and only if

$$
\frac{\sigma^{4}}{c}>\frac{M P V_{1}\left(\hat{t}_{1}\right)}{\sigma^{2}\left(1-\hat{t}_{1}\right)^{2}} .
$$

The right-hand side depends only on $n, \beta$, and $\lambda$. Call it $x_{1}$. Now overinvestment occurs if and only if $\sigma^{4} / c>x_{1}$.

The proof of (ii) goes along the same lines.

\subsection{Comparative statics}

Proof of Lemma 6

(i)

$$
E_{j}^{\prime}\left(t_{i}\right)=\frac{\rho_{i} \rho_{j}\left(\beta^{2}+2 \rho_{i}^{2}\left(\rho_{j}^{2}-\beta\right)+2 \rho_{j}^{2}\left(\rho_{i}^{2}-\beta\right)\right)}{\left(\rho_{i}\left(2 \rho_{i} \rho_{j}-\beta\right)+t_{i} \beta\left(\rho_{i}-\rho_{j}\right)\right)^{2}}>0 .
$$

From (21) it easily follows that $E_{j}^{\prime \prime}\left(t_{i}\right)<0$ and, hence, $E_{j}^{\prime}\left(t_{i}\right) \leq E_{j}^{\prime}(0)<1$.

(ii) Trivial.

(iii)

$$
R_{j}^{\prime}\left(t_{i}\right)=\frac{-2 \rho_{i} \rho_{j}^{2} \beta \sqrt{c}\left[2 \rho_{i} \beta \sqrt{c}+\sigma^{2}\left(2 \rho_{i}^{2}-\beta\right)\right]}{\left[t_{i} \beta^{2} \sqrt{c}-\beta \sigma^{2} \rho_{i}\left(1-t_{i}\right)\right]^{2}}<0
$$

$$
\begin{gathered}
\frac{\partial R_{j}\left(t_{i}\right)}{\partial \sqrt{c}}=\frac{-2\left(1-t_{i}\right) \beta \rho_{i} \rho_{j}^{2}\left(2 \rho_{i}^{2}-t_{i} \beta\right)}{\left[t_{i} \beta^{2} \sqrt{c}-\beta \sigma^{2} \rho_{i}\left(1-t_{i}\right)\right]^{2}}<0 . \\
\frac{\partial R_{j}\left(t_{i}\right)}{\partial \rho_{i}}=\frac{2 \beta \rho_{j}^{2} \sqrt{c}\left[4 \rho_{i} t_{i} \beta \sqrt{c}-2 \rho_{i}^{2} \sigma^{2}\left(1-t_{i}\right)-\sigma^{2}\left(1-t_{i}\right) t_{i} \beta\right]}{\left[t_{i} \beta^{2} \sqrt{c}-\beta \sigma^{2} \rho_{i}\left(1-t_{i}\right)\right]^{2}}<0 .
\end{gathered}
$$

The inequality follows from $\sigma^{2}\left(1-t_{i}\right) \geq 2 \rho_{i} \sqrt{c}$ (on the relevant range, i.e. where $R_{j}\left(t_{i}\right) \geq$ $0)$.

To see that $R_{j}\left(t_{i}\right)$ increases with increases in $\lambda_{j}$ note that $R_{j}\left(t_{i}\right)$ can be written as the product of $\rho_{j}^{2}$ and a factor that does not depend on $\lambda_{j}$.

Finally,

$$
\frac{\partial R_{j}\left(t_{i}\right)}{\partial \sigma^{2}}=\frac{2\left(1-t_{i}\right) \beta \rho_{i} \rho_{j}^{2} \sqrt{c}\left(2 \rho_{i}^{2}-t_{i} \beta\right)}{\left[t_{i} \beta^{2} \sqrt{c}-\beta \sigma^{2} \rho_{i}\left(1-t_{i}\right)\right]^{2}}>0 .
$$

(v) As (iv).

(vi) $R_{j}(t)$ is the product of two positive factors (at least on the relevant range) which are both decreasing in $\beta$. Namely,

$$
R_{j}(t)=\frac{2 \rho_{j}^{2}}{\beta} \times \frac{\sigma^{2} \rho_{i}(1-t)-2 \rho_{i}^{2} \sqrt{c}}{\sigma^{2} \rho_{i}(1-t)-t \beta \sqrt{c}}
$$

and

$$
\frac{\partial}{\partial \beta}\left(\frac{2 \rho_{j}^{2}}{\beta}\right)=\frac{-2 \lambda_{j}}{\beta^{2}}<0
$$


while

$$
\begin{aligned}
\frac{\partial}{\partial \beta} & \left(\frac{\sigma^{2} \rho_{i}(1-t)-2 \rho_{i}^{2} \sqrt{c}}{\sigma^{2} \rho_{i}(1-t)-t \beta \sqrt{c}}\right)= \\
& \frac{-\sigma^{2}(1-t)^{2} \rho_{i} \sqrt{c}-\sigma^{2}(1-t) t \beta \sqrt{c} /\left(2 \rho_{i}\right)-2\left(\rho_{i}^{2}-\beta\right) t c}{\left(\sigma^{2} \rho_{i}(1-t)-t \beta \sqrt{c}\right)^{2}}<0 .
\end{aligned}
$$

(vii) Note that $(\partial / \partial \beta)\left(\rho_{k}\right)=1 /\left(2 \rho_{k}\right)$. It can be verified that

$$
\begin{aligned}
& \operatorname{sign}\left(\frac{\partial E_{j}(t)}{\partial \beta}\right)= \\
& \quad \operatorname{sign}\left\{( \rho _ { i } - \rho _ { j } ) \left[\frac{\rho_{j}}{\rho_{i}}\left(\rho_{i}-\rho_{j}\right) t \beta+\frac{\rho_{i}^{2}}{\rho_{j}}\left(2 \rho_{i} \rho_{j}-\beta\right)+\frac{\rho_{i}}{\rho_{j}}\left(\rho_{i}-\rho_{j}\right) t \beta+\right.\right. \\
& \quad-\rho_{i}\left(2 \rho_{i} \rho_{j}-\beta\right)+t\left(2 \rho_{i} \rho_{j}-\beta\right)^{2} \frac{\rho_{i}+\rho_{j}}{2 \rho_{i} \rho_{j}}+t^{2} \frac{\beta}{2 \rho_{j}}\left(2 \rho_{i} \rho_{j}-\beta\right)+ \\
& \quad t^{2} \beta \rho_{j}\left(\frac{\rho_{j}}{\rho_{i}}+\frac{\rho_{i}}{\rho_{j}}-1\right)-2 \rho_{i} \rho_{j}\left(\rho_{i}-\rho_{j}\right) t-t^{2} \rho_{j}\left(2 \rho_{i} \rho_{j}-\beta\right)+ \\
& \left.\left.\quad t^{2} \frac{\beta}{2 \rho_{i}}\left(2 \rho_{i} \rho_{j}-\beta\right)-2 \rho_{i}^{2} \rho_{j}\left(\frac{\rho_{j}}{\rho_{i}}+\frac{\rho_{i}}{\rho_{j}}-1\right)\right]\right\}
\end{aligned}
$$

We may now multiply the term between the square brackets by $2 \rho_{i} \rho_{j}$ and write it as a quadratic expression in $t$. Note that $E_{j}(1) \equiv 1$ so that the expression between brackets equals zero for $t=1$. Using that $\lambda_{i}>\lambda_{j}$ we find that

$$
\frac{\partial E_{j}(t)}{\partial \beta}<0
$$

if and only if

$$
(t-1)(A t+B)<0
$$

where,

$$
A=4 \rho_{i}^{2} \rho_{j} \beta+2 \rho_{j}^{3} \beta-\rho_{i} \beta^{2}-\rho_{j} \beta^{2}-4 \rho_{i}^{2} \rho_{j}^{3}+2 \rho_{i} \rho_{j}^{2} \beta
$$

and

$$
B=2 \rho_{i}^{3} \beta-2 \rho_{i}^{2} \rho_{j} \beta+4 \rho_{i}^{2} \rho_{j}^{3} .
$$

The statement (vii) follows now from the observation that $B>0$ and that if $A<0$ then $-B / A>1$.

\section{References}

Chang, C.-H., AND LeE C.-W. J. (1992). "Information Acquisition as Business Strategy," Southern Economic Journal, 58, 750-761.

Daughety, A.F. And Reinganum, J.F. (1992). “Asymmetric Information Acquisition and Behavior in Role Choice Models: An Endogenously Generated Signalling Game," mimeo, University of Iowa. 
GaL-Or, E. (1985). "Information Sharing in Oligopoly," Econometrica, 53 329-343.

Hurkens, S. And Vulkan, N. (1995). "Information Acquisition and Entry," mimeo $\mathrm{UCL}$ and UPF.

HwANG, H. (1993). "Optimal Information Acquisition for Heterogeneous Duopoly Firms," Journal of Economic Theory, 59, 385-402.

HWAng, H. (1995). "Information Acquisition and Relative Efficiency of Competitive, Oligopoly, and Monopoly Markets," International Economic Review, 36, 325-340.

Levine, P. And Ponssard, J.-P. (1977). "The Values of Information in Some Nonzero Sum Games," International Journal of Game Theory, 6, 221-229.

Li, L., McKelvey, R.D., And Page, T. (1987). "Optimal Research for Cournot Oligopolists," Journal of Economic Theory, 42, 140-166.

Matthews, S. (1984). "Information Acquisition in Discriminatory Auctions," in: Bayesian Models in Economic Theory, eds. M. Boyer and R.E. Kihlstrom. Elsevier.

Milgrom, R. (1981). "Rational Expectations, Information Acquisition and Competitive Bidding," Econometrica, 49, 921-944.

OCKEnfels, P. (1989). Informationsbeschaffung auf homogenen Oligopolmärkten: eine spieltheoretische Analyse. Physica-Verlag Heidelberg.

Ponssard, J.-P., (1979). “The Strategic Role of Information on the Demand Function in an Oligopolistic Market," Management Science, 25, 243-250.

VIVES, X. (1988). “Aggregation of Information in Large Cournot Markets," Econometrica, 56, 851-876. 